\section{Scanning Electron Microscopic Study of Modified Chloroplasts in Senescing Broccoli Florets}

\author{
Hirofumi Terai ${ }^{1}$ and Alley E. Watada \\ Horticultural Crops Quality Laboratory, Beltsville Agricultural Research \\ Center, Agricultural Research Service, U.S. Department of Agriculture, \\ Beltsville, MD 20705-2350
}

\author{
Charles A. Murphy and William P. Wergin \\ Nematology Laboratory, Beltsville Agricultural Research Center, Agricultural \\ Research Service, U.S. Department of Agriculture, Beltsville, MD 20705-2350
}

Additional index words. broccoli, ultrastructure, chloroplast, SEM, TEM, freeze-fracture

\begin{abstract}
Structural changes in chloroplasts of broccoli (Brassica oleracea L., Italica group) florets during senescence were examined using light microscopy, scanning electron microscopy (SEM) with freeze-fracture technique, and transmission electron microscopy (TEM) to better understand the process of chloroplast degradation, particularly at the advanced stage of senescence. Light microscopy revealed that chloroplasts, which initially were intact and green, became obscure in shape, and their color faded during senescence. Small, colored particles appeared in cells as the florets approached the final stage of senescence and became full- to dark-yellow in color. Scanning electron microscopy showed that stroma thylakoids in the chloroplast initially were parallel to each other and grana thylakoids were tightly stacked. As senescence advanced, the grana thylakoids degenerated and formed globules. The globules became larger by aggregation as senescence progressed, and the large globules, called "thylakoid plexus," formed numerous vesicles. The vesicles ultimately were expelled into the cytosol, and the light microscope revealed many colored particles in the senescent cells. These results indicate that the degradation of chloroplasts in broccoli florets progresses systematically, with the final product being colored particles, which are visible in yellow broccoli sepal cells.
\end{abstract}

Structural changes in chloroplasts have been studied with both scanning electron microscopy (SEM) (Blackmore et al., 1984; Kulandaivelu and Gnanam, 1985; Larsson et al., 1977; Mohapatra and Johnson, 1976; Tuquet and Newman, 1980) and transmission electron microscopy (TEM). Plant materials studied with TEM have included senescing leaves of wheat (Triticum aestivum L.) (Hurkman, 1979), Phaseolus (Barton, 1966; Ronning et al., 1991), spinach (Spinacia oleracea L.) (Mine et al., 1992), and Betula (Dodge, 1970), ripening fruit of Capsicum (Spurr and Harris, 1968) and mango (Mangifera indica L.) (Parikh et al., 1990), both developing and senescing fruit of normal and mutant tomato (Lycopersicum esculentum Mill.) (Simpson et al., 1976), and ethylenetreated fruit of Satsuma mandarin (Citrus reticulata Blanco) (Shimokawa et al., 1978)

\footnotetext{
Received for publication 24 Sept. 1998. Accepted for publication 5 May 1999. Use of company or product name by the U.S. Dept. of Agriculture does not imply approval or recommendation of the product to the exclusion of others which also may be suitable. The cost of publishing this paper was defrayed in part by the payment of page charges. Under postal regulations, this paper therefore must be hereby marked advertisement solely to indicate this fact.

${ }^{1}$ To whom reprint requests should be addressed. Present address: Faculty of Agriculture, Kobe University, Rokkodai-cho Nada-ku, Kobe 657-8501, Japan. E-mail address: terai@kobe-u.ac.jp
}

and leaves of morning glory [Ipomoea nil (L.) Roth] (Toyama, 1980).

Gepstein (1988) postulated the following sequential changes in senescing chloroplasts: 1) stroma thylakoids lost integrity; 2) chloroplasts increased in volume, and in some plants, the number of osmiophilic granules increased; 3) grana thylakoids swelled, then gradually disintegrated; and 4) the chloroplast envelope retained its integrity as the tissue entered near the final stage of senescence. These postulations were based on observations made by Gepstein and others on chloroplasts at various stages of senescence, but not at the final stage.

A better and complete understanding of ultrastructural changes in chloroplasts is obtained by two-dimensional analysis with TEM and by three-dimensional analysis with SEM using a freeze-fracture technique. Barnes and Blackmore (1984) used SEM with freezefracture technique to examine the contents of chloroplasts but concentrated on specific stage rather than following sequential changes during senescence.

We were interested in the effects of various postharvest treatments on maintenance of green color in broccoli florets, but needed to know the ultrastructural changes of chloroplasts during senescence to seek effective treatments. To our knowledge, no one has reported on the ultrastructural changes of chloroplasts in these tissues. Fruit or leaf blades were used in the studies cited above
In this study, we examine and discuss the ultrastructural changes in chloroplasts of broccoli florets during senescence, as observed with TEM, SEM with freeze fracture, and light microscopy.

\section{Materials and Methods}

Fresh, green broccoli heads were obtained from the Wholesale Distribution Center, Jessup, Md. A series of florets was excised from the head and allowed to senesce to the following stages for analysis: non-yellow $=$ no yellow florets; slight-yellow = lighter green and slight yellow florets visible; half-yellow = green and yellow florets present in about equal amounts; full-yellow $=$ most of the florets light yellow; dark-yellow $=$ all florets dark yellow.

For analysis by light microscopy, the heads were placed in a perforated polyethylene bag at $20{ }^{\circ} \mathrm{C}$. Florets were detached daily from a single head to obtain successive stages of senescence, and immersed in a $2 \%$ warm agar solution in a petri dish. The agar containing the florets was allowed to gel in a refrigerator and sliced into 5- $\mu \mathrm{m}$-thick sections. For SEM and TEM observations, short-stemmed branchlets weighing $\approx 30 \mathrm{~g}$ were excised from a head, placed individually in $100-\mathrm{mL}$ beakers containing sufficient water to cover the base of the stem, and stored at $1^{\circ} \mathrm{C}$. One beaker containing the branchlets was transferred daily from 1 to $20^{\circ} \mathrm{C}$ for $6 \mathrm{~d}$ to obtain florets of different stages of senescence. The sepals were removed from the florets and prepared for examination by electron microscopy.

For observation of freeze-fractured specimens, sepals of florets were fixed in $2 \%(\mathrm{w} / \mathrm{v})$ $\mathrm{OsO}_{4}$ in phosphate buffer $(0.1 \mathrm{M}, \mathrm{pH}$ 7.4) for 2 h. Following fixation, one set of specimens was rinsed with the phosphate buffer and dehydrated through a gradient series of ethanol (50\% to $70 \%)$. Next, the specimens were frozen in liquid nitrogen and fractured with a scalpel. The fractured specimens were rehydrated back to phosphate buffer through a gradient series of ethanol from $70 \%$ to $50 \%$. A second set of specimens fixed with $\mathrm{OsO}_{4}$ was dehydrated with a gradient series of solutions of dimethyl sulfoxide (DMSO) (25\% to $50 \%$ ) and fractured with DMSO instead of ethanol to minimize the formation of ice crystals, which would cause mechanical disruption of the tissues. However, DMSO residue interferes with extraction of the proteinaceous matrix of plastids, which restricts accurate observation and evaluation of the fine structural features of the thylakoid membranes. Thus, micrographs from both procedures were used for analysis and interpretation of data.

The specimens fractured with ethanol or DMSO series were transferred to $0.1 \% \mathrm{OsO}_{4}$ for $8 \mathrm{~d}$. The $\mathrm{OsO}_{4}$ solution was replaced daily with a fresh supply. Next, specimens were treated in sequence with $1 \% \mathrm{OsO}_{4}$ for $1 \mathrm{~h}, 1 \%$ tannic acid for $2 \mathrm{~h}$, and with a second solution of $1 \% \mathrm{OsO}_{4}$ for $1 \mathrm{~h}$ and dehydrated through a gradient series of ethanol (50\% to $100 \%)$. To minimize tissue distortion that is associated with air drying, the samples were placed in an Autosamdri-810 critical point drying appara- 
tus (Tousimis Research Corp., Rockville, Md.). In this dryer, the ethanol was replaced with liquid carbon dioxide that was subsequently raised to its critical point $\left(31^{\circ} \mathrm{C}\right.$ and $7.3 \times 10^{6}$ $\mathrm{Pa}$ ) where the fluid and vapor phases are indistinguishable. Next, the dryer was vented to room pressure, and the samples were removed and mounted on aluminum stubs that were sputter-coated with $\mathrm{Au} / \mathrm{Pd}$ and then inserted into a Hitachi S-570 SEM (Hitachi Scientific Instruments, Mountain View, Calif.) for observation.

For TEM observation, small segments $(\approx 0.5$ $\mathrm{mm}$ square) of sepals were fixed in $3 \%$ glutaraldehyde in phosphate buffer (0.1 м pH 7.4), rinsed with the same buffer, and postfixed in $2 \%$ buffered $\mathrm{OsO}_{4}$ for $2 \mathrm{~h}$. Following fixation, the segments were rinsed with the buffer, dehydrated through a gradient series of ethanol $(20 \%$ to $100 \%)$, and embedded in Spurr' s low-viscosity epoxy resin (Spurr, 1969). Sections were cut on an Ultracut ultramicrotome (AO Scientific Instruments, Buffalo, N.Y.), stained with $2 \%$ uranyl acetate and Reynold's lead citrate and examined in a Hitachi $\mathrm{H}-500 \mathrm{H}$ TEM (Hitachi Scientific Instruments).

\section{Results and Discussion}

The broccoli florets senesced and changed color from non-yellow to dark-yellow during the $6 \mathrm{~d}$ at $20^{\circ} \mathrm{C}$. Examination by light microscopy indicated that the chloroplasts were green and intact at the non-yellow stage (Fig. 1a), less visible at the slight-yellow stage (Fig. 1b), and indistinct at the half-yellow stage because they were masked with pigments (Fig. 1c). At the full- to dark-yellow stage, small green or yellow-to-brown particles were seen dispersed in the cells (Fig. 1d). With SEM, chloroplasts were recognizable at the non-yellow stage (Fig. 2a), the slight-yellow stage (Fig. 2b), and the half-yellow stage (Fig. 2c-f), when considerable architectural changes had taken place. Chloroplasts underwent significant structural changes during the half-yellow to full-yellow stage with the formation of colored particles.

At the non-yellow stage, SEM observations indicated that stroma thylakoids generally were oriented parallel to the long axis of the chloroplasts and the grana were stacked compactly and tightly (Fig. 2a). At the slightyellow stage, the chloroplasts were more round and the thylakoid membranes were less organized; the grana thylakoids were less distinct and the stacks showed a loss of their orderly structure (Fig. 2b). At the half-yellow stage, discrete grana thylakoids were not distinguishable, and dense globules appeared in the stroma (Fig. 2c).

As the stroma and grana thylakoid membranes became disorganized, globules appeared in the stroma and many of the globules fused together. Globules are products of the disorganized thylakoid membranes, which contain lipids and carotenoids (Barton, 1966; Matile, 1992). Although Thomson (1966) found that there was an increase in size and number of large globules in flavedo of 'Valencia' oranges [Citrus sinensis (L.) Osbeck], Dodge (1970) found that size, but not the number, increased during the autumnal senescence of Betula leaves. We found that the globules gathered and then fused together and became larger at the half-yellow stage (Fig. 2d).

The fused large globules, which have been referred to as "thylakoid plexus" (Harris and Spurr, 1969; Spurr and Harris, 1968), appeared after the grana and stroma thylakoid system had undergone considerable changes. The thylakoid plexus and vesicles, which look like empty granular bags in SEM micrographs, were noted in the senescent chloroplasts (Fig. $2 \mathrm{~d}$ and $\mathrm{e})$. Vesicles appeared on the periphery of thylakoid plexus at the half-yellow stage (Fig. $2 \mathrm{~d}$ and e). The thylakoid plexus appeared to have formed many vesicles. The threedimensional image of thylakoid plexus, as observed with SEM, indicated the structure to be spheres of variable size (Fig. 2d), and that the mass had generated many vesicles.

The vesicles were formed from the thylakoid plexus, which was the product of disorga-
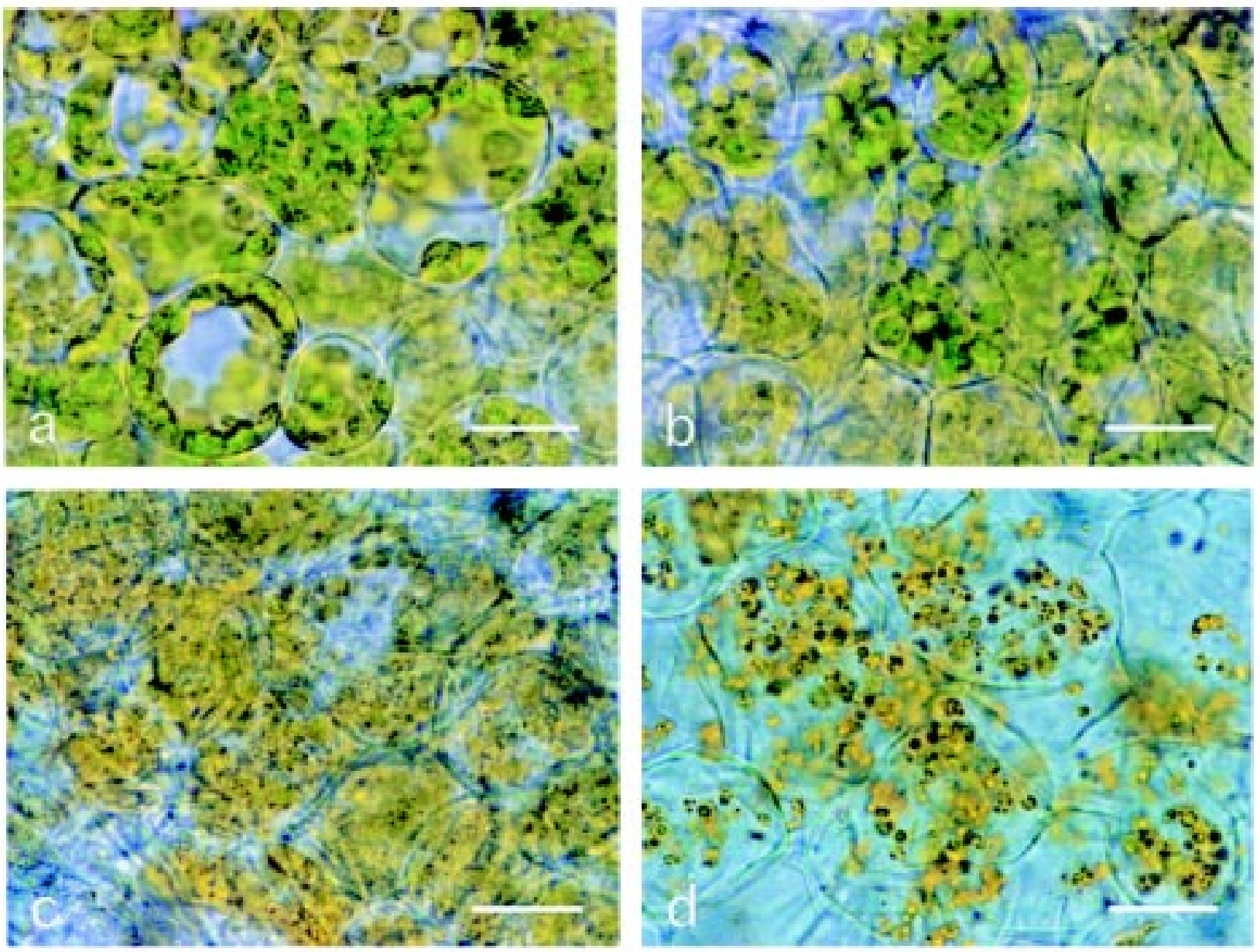

Fig. 1. Light micrographs of chloroplasts in the sepal cells of broccoli florets during storage at $20^{\circ} \mathrm{C}$. The chloroplasts were: (a) green and intact at the non-yellow stage; (b) less distinct at the slight-yellow stage; (c) not distinct at the half-yellow stage. (d) At the full- to dark-yellow stage, only small yellow/brown colored particles appeared in the cells. All scales indicate $20 \mu \mathrm{m}$. 

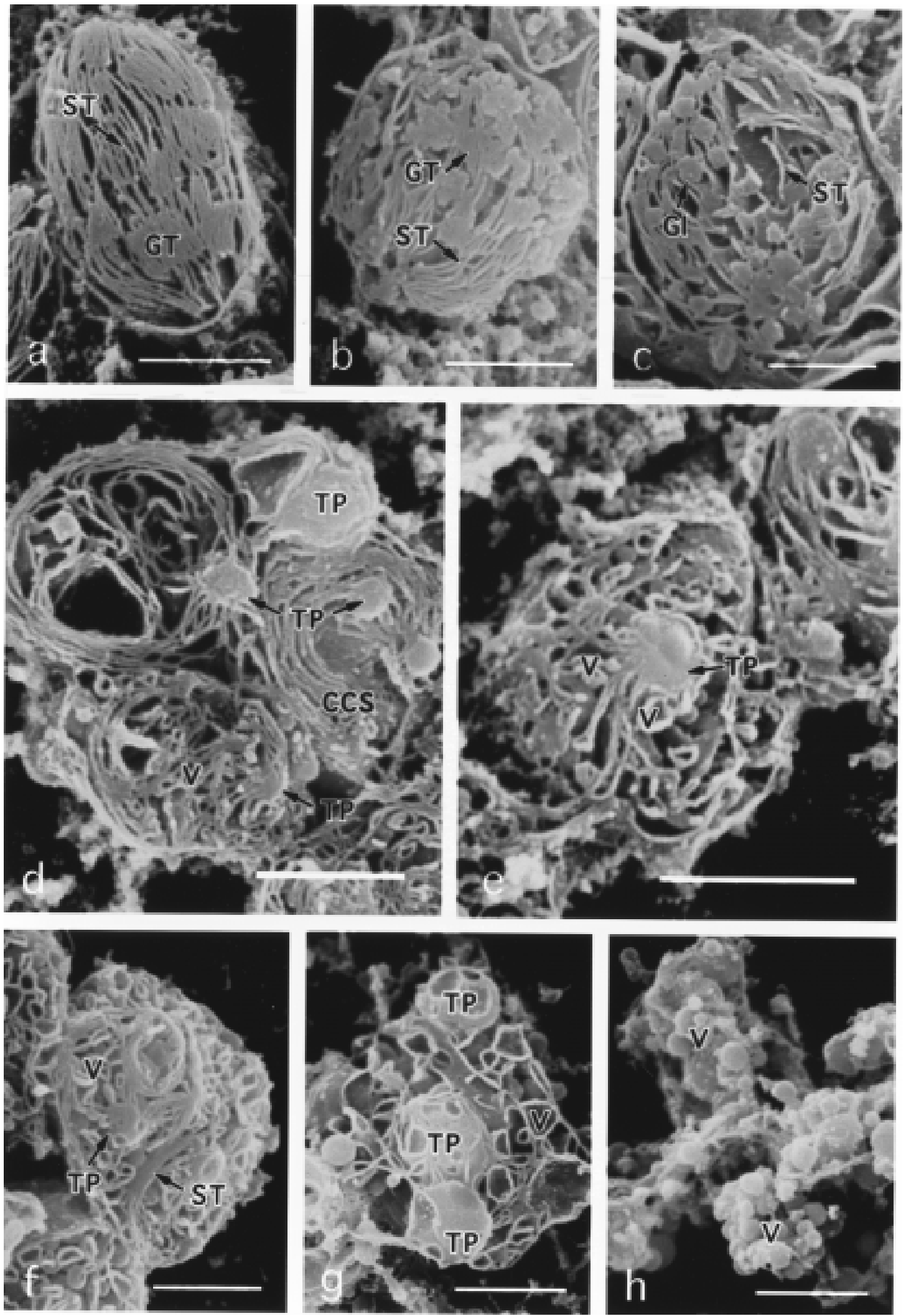

Fig. 2. Scanning electron micrographs of various stages of chloroplasts in the sepal cells of broccoli florets during storage at $20^{\circ} \mathrm{C}$. In the preparation of specimens for SEM, sepals of broccoli florets were fractured in liquid nitrogen with ethanol after fixation with $\mathrm{OsO}_{4}$ as described in the text. (a) Non-yellow stage: stroma thylakoids (ST) oriented parallel to the long axis of chloroplast and the grana thylakoids (GT) stacked compactly and tightly. (b) Slight-yellow stage: chloroplasts more rounded, orientation of stroma thylakoids (ST) beginning to change and stacks of grana thylakoids (GT) becoming less distinct. (c) Halfyellow stage: grana stacks no longer discrete, although globular bodies (Gl) have appeared and stroma thylakoids (ST) remain. (d) Half-yellow stage: complexes that probably correspond to "thylakoid plexus" (TP) and small vesicles (V) apparent, and concentric circular structure (CCS) apparently formed from stroma thylakoids. (e) Half-yellow stage: many small vesicles (V) at the periphery of the thylakoid plexus (TP). (f) Half-yellow stage: the inside of a senescing chloroplast with numerous vesicles (V), disorganized stroma thylakoid (ST), and thylakoid plexus (TP). (g) Full-yellow stage: vesicles (V) and thylakoid plexus (TP) prominent. (h) Dark-yellow stage: integrity of the chloroplast completely lost, leaving only aggregations of spherical bodies that appear to be vesicles (V). All scales indicate $1 \mu \mathrm{m}$. 


\section{Postharvest Biology \& Technology}
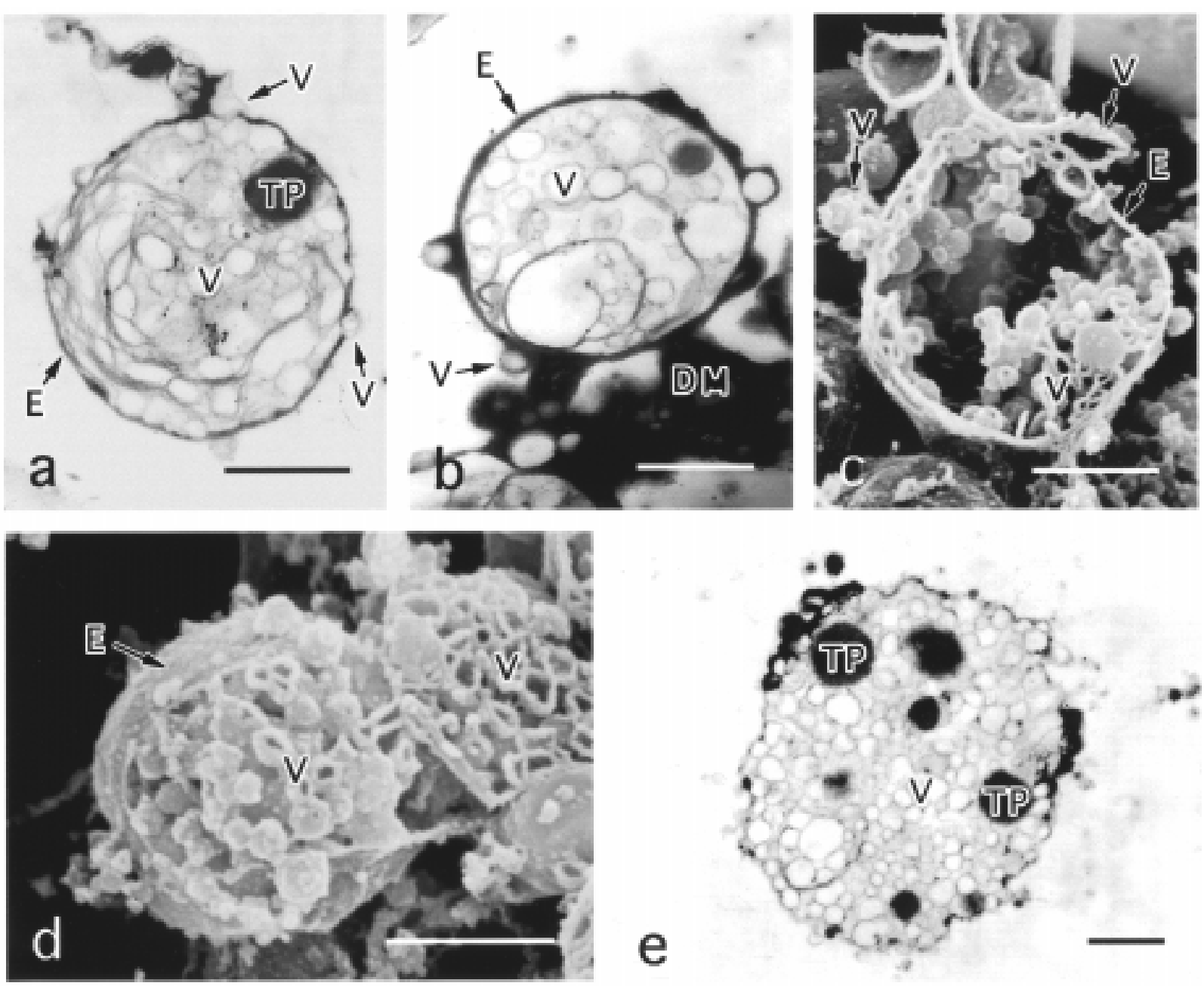

Fig. 3. Scanning and transmission electron micrographs of chloroplasts at the full-yellow stage of broccoli floret sepals during storage at $20^{\circ} \mathrm{C}$. In the preparation of specimens for SEM, sepals of broccoli florets were fractured in liquid nitrogen with DMSO after fixation with $\mathrm{OsO}_{4}$ as described in the text. (a) The "thylakoid plexus" (TP) has formed vesicles (V) but few vesicles appear to have emerged through the envelope (E). (b) Few vesicles (V) with dense material (DM) outside of the chloroplast envelope (E), and the inside space of chloroplast conspicuous. (c) Decrease in density of vesicles $(\mathbf{V})$ in chloroplasts with few vesicles on the outside of the envelope $(\mathbf{E})$. (d) Some vesicles $(\mathbf{V})$ on the outside of the senescing chloroplast, which lacks a complete envelope (E). (e) Many vesicles (V) and some thylakoid plexus (TP) noted in the senescent chloroplast, no distinct envelope or double membrane apparent. All scales indicate $1 \mu \mathrm{m}$.

nized and disintegrated thylakoid membrane. This contrasts with the findings of Hurkman (1979), who reported that the vesicles formed from the distention of grana and stroma thylakoids in the chloroplast of senescent primary wheat leaves. Formation of the vesicles may differ among plant species or structure.

Concentric circles of thylakoid membrane appeared with thylakoid plexus (Fig 2d), as previously observed in mutants of maize (Zea mays L.) (Bachmann et al., 1967), in tobacco (Nicotiana tabacum L.) leaves infected by virus (Ehara and Misawa 1975), in tangerine tomato (Rosso, 1967), and in Capsicum fruit (Spurr and Harris, 1968). How the concentric circles are formed is not known, but they may result from stacking and rolling of stroma thylakoid after grana disintegrate and change to thylakoid plexus.

After their formation in the senescing chloroplast at the half-yellow stage (Fig. 2f), the vesicles became enlarged like small balloons; the connection between the vesicles appeared to be lost at the full-yellow stage (Fig. 2g). Eventually, at the dark-yellow stage (Fig. 2h), the vesicles and other structures including thylakoid plexus looked like clusters of grapes.

For better understanding the emergence of vesicles and globules from senescent chloroplast to cytosol at the full-yellow stage, additional observations were conducted using TEM and SEM (Fig. 3). Emergence of the particles on the outside of senescent chloroplasts may have resulted by either or both of the following: 1) vesicles and globules emerged through the envelope of the senescent chloroplast to the cytosol, which might result in the decrease in density of these particles in chloroplasts as shown in micrographs (Fig. 3a-c); and/or 2) the envelope disappeared partially at the site where the vesicles and globules were attached (Fig. 3d). With the destruction of the envelope, many globules and vesicles were exposed to the cytosol (Fig. 3e). Hurkman (1979) presented TEM micrographs, which indicated a discontinuous envelope membrane of senescent chloroplasts containing numerous vesicles and globules. These globules and vesicles, which contained carotenoids and other pigments, were found in the senescent cells of broccoli sepals (Fig. 1d, 2h).

In summary, the chloroplasts in broccoli florets underwent organized degradative and developmental changes during senescence. The grana and stroma thylakoids became disorganized and disintegrated, and subsequently large globules identified as thylakoid plexus were formed, which produced vesicles (the colored particles visible by light microscopy). The disorganization and disintegration of grana and stroma thylakoids need to be prevented to keep the florets green. To control these changes, biochemical studies are needed to identify the enzymes and reactions involved.

\section{Literature Cited}

Bachmann, M.D., D.S. Robertson, C.C. Bowen, and I.C. Anderson. 1967. Chloroplast development in pigment deficient mutants of maize. I. Structural anomalies in plastids of allelic mutants at the $\mathrm{W}_{3}$ locus. J. Ultrastruct. Res. 21:41-60.

Barnes, S.H. and S. Blackmore. 1984. Scanning electron microscopy of chloroplast ultrastructure. Micron Microscopica Acta 15:187-194.

Barton, R. 1966. Fine structure of mesophyll cells in senescing leaves of Phaseolus. Planta 71:314-325.

Blackmore, S., S.H. Barnes, and D. Claugher. 1984 Scanning electron microscopy of cytoskeletal components in Aucuba japonica leaves. J. 
Ultrastruct. Res. 86:215-219.

Dodge, J.D. 1970. Changes in chloroplast fine structure during the autumnal senescence of Betula leaves. Ann. Bot. 34:817-824.

Ehara, Y. and T. Misawa. 1975. Occurrence of abnormal chloroplasts in tobacco leaves infected systemically with the ordinary strain of cucumber mosaic virus. Phytopath. Z. 84:233-252.

Gepstein, S. 1988. Photosynthesis, p. 85-109. In: L.D. Noodén and A.C. Leopold (eds.). Senescence and aging in plants. Academic Press, San Diego.

Harris, W.M. and A.R. Spurr. 1969. Chromoplasts of tomato fruits. II. The red tomato. Amer. J. Bot. 56:380-389.

Hurkman, W.J. 1979. Ultrastructural changes of chloroplasts in attached and detached, aging primary wheat leaves. Amer. J. Bot. 66:64-70.

Kulandaivelu, G. and A. Gnanam. 1985. Scanning electron microscopic evidence for a budding mode of chloroplast multiplication in higher plants. Physiol. Plant. 63:299-302.

Larsson, C., B. Andersson, and G. Roos. 1977. Scanning electron microscopy of different populations of chloroplasts isolated by phase partition. Plant Sci. Lett. 8:291-298.
Matile, P. 1992. Chloroplast senescence, p. 413440. In: N.R. Baker and H. Thomas (eds.). Topics in photosynthesis, Vol. 12. Crop photosynthesis: Spatial and temporal determinants. Elsevier, Amsterdam.

Mine, H., K. Masuhara, and S. Tamura. 1992. Changes in fine structure of chloroplasts in stored spinach leaves. J. Home Econ. Jpn. 43:23-29.

Mohapatra, S.C. and W.H. Johnson. 1976. Microscopic studies on bright leaf tobacco. V. Scanning electron microscopic observations on chloroplast structure and distribution. Trans. Amer. Microsc. Soc. 95:228-232.

Parikh, H.R., G.M. Nair, and V.V. Modi. 1990 Some structural changes during ripening of mangoes (Mangifera indica var. Alphonso) by abscisic acid treatment. Ann. Bot. 65:121-127.

Ronning, C.M., J.C. Bouwkamp, and T. Solomos. 1991. Observations on the senescence of a mutant non-yellowing genotype of Phaseolus vulgaris L. J. Expt. Bot. 42:235-241.

Rosso, S.W. 1967. An ultrastructural study of the mature chromoplasts of the tangerine tomato (Lycopersicon esculentum var. 'Golden Jubilee'). J. Ultrastruct. Res. 20:179-189.

Shimokawa, K., A. Sakanoshita, and K. Horiba.
1978. Ethylene-induced changes of chloroplast structure in Satsuma mandarin (Citrus unshiu Marc.). Plant Cell Physiol. 19:229-236.

Simpson, D.J., M.R. Baqar, W.B. McGlasson, and T.H. Lee. 1976. Changes in ultrastructure and pigment content during development and senescence of fruits of normal and rin and nor mutant tomatoes. Austral. J. Plant Physiol. 3:575-587.

Spurr, A.R. 1969. A low-viscosity epoxy resin embedding medium for electron microscopy. J. Ultrastruct. Res. 26:31-43.

Spurr, A.R. and W.M. Harris. 1968. Ultrastructure of chloroplasts and chromoplasts in Capsicum апnиит I. Thylakoid membrane changes during fruit ripening. Amer. J. Bot. 55:1210-1224.

Thomson, W.W. 1966. Ultrastructural development of chromoplasts in Valencia oranges. Bot. Gaz. 127:133-139.

Toyama, S. 1980. Electron microscope studies on the morphogenesis of plastids. X. Ultrastructural changes of chloroplasts in morning glory leaves exposed to ethylene. Amer. J. Bot. 67:625-635.

Tuquet, C. and D.W. Newman. 1980. Aging and regreening in soybean cotyledons. 1. Ultrastructural changes in plastids and plastoglobuli. Cytobios 29:43-59. 